\title{
Radiotherapy Interruption in Cancer Patients: Rates and Causes at Ahvaz Golestan Hospital
}

\author{
Sasan Razmjoo ${ }^{1}$, Negar Haghi², Mohammad Javad Tahmasebi Birgani ${ }^{3}$, Shole \\ Arvandi', Seyed Mohammad Hosseini ${ }^{4}$, Hodjatollah Shahbazian ${ }^{4}$, Mohammad \\ reza javan ${ }^{5}$
}

\begin{abstract}
${ }^{1}$ Assistant Professor of Radiotherapy and Oncology, Department of Clinical Oncology, Ahvaz Jundishapur University of Medical Sciences, Ahvaz, Iran. ${ }^{2}$ Medical Student, School of Medicine, Ahvaz Jundishapur University of Medical Sciences, Ahvaz, Iran. ${ }^{3}$ Professor of Medical Physics, Department of Clinical Oncology, Ahvaz Jundishapur University of Medical Sciences, Ahvaz, Iran. ${ }^{4}$ Associate Professor of Radiotherapy and Oncology, Department of Clinical Oncology, Ahvaz Jundishapur University of Medical Sciences, Ahvaz, Iran. ${ }^{5}$ High Institute for Research \& Education in Transfusion Medicine IBTO.
\end{abstract}

\begin{abstract}
Introduction: Radiotherapy is one of the most common cancer treatment modalities. The goal of this study was to establish the rates and causes of radiotherapy interruptions in cancer patients at Ahvaz Golestan Hospital. Methods: In this retrospective study, the data were collected during period from 2012 to 2013, from cancer patients who received radiation therapy at Golestan Hospital. Demographic characteristics and radiotherapy interruption frequency and reasons were reviewed and analyzed. Results: Among 1476 cases, $70.7 \%$ of patients had no radiotherapy interruption. The most common cause of treatment interruption was equipment damages and/or maintenance in $29.5 \%$ of patients. There were statistically significant relations between radiotherapy interruption with site of cancer $(\mathrm{P}=0.014)$ and living place of patients $(\mathrm{P}=0.006)$, respectively. Conclusion: Generally, treatment interruption at our center was not much higher than other centers; however, the most common cause of treatment interruption was equipment damages and/or maintenance, which was higher than most centers.
\end{abstract}

Keywords: Radiotherapy- interruption- Cancer

Asian Pac J Cancer Care, 5 (1), 33-36

\section{Introduction}

Cancer is one of the most common causes of death all over the world which is spreading fast. Different types of treatment modalities are used in different types of cancers; such as surgery, chemotherapy, and radiotherapy [1]. Radiotherapy is based on exposing malignant cells to ionizing radiation, which can lead to death of these cells [2]. The ideal goal of radiotherapy is the delivery of more doses to tumor cells and less doses to the surrounding normal tissues to produce a high probability of tumor control whilst causing minimal normal tissue complications [3].

A conventional course of radiotherapy is scheduled for five days per week over several weeks; however, interruptions in this course may occur and unplanned gaps are a common occurrence. These gaps and interruptions in
Submission Date: 11/18/2019 Acceptance Date: 01/12/2020

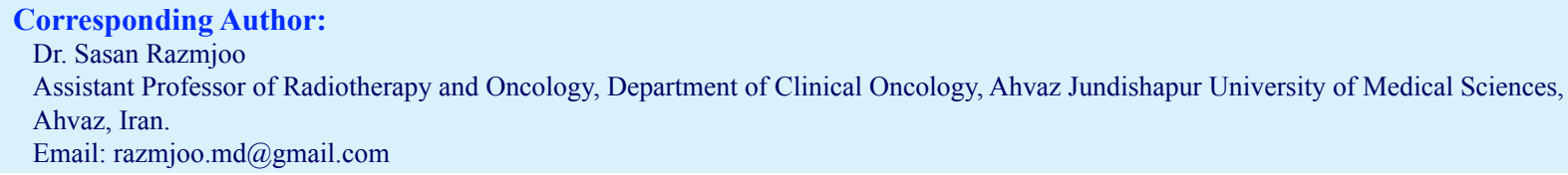

treatment regime may lead to the repopulation of tumor clonogens [4]. In particular for the head and neck cancers for which the evidence is the strongest for accelerated repopulation of clonogenic tumor cells, it is estimated that each one and seven days interruptions in treatment course result in $0.68-1.4 \%$ and $14-20 \%$ reduction in the local control rate, respectively [5-6-7].

Completion of radiotherapy schedule has special importance for increasing tumor control probability and reducing the probability of tumor progression and/ or recurrence [8-9-10]. Thus, one of the main goals in radiation therapy is the delivery of the total dose without any interruptions or prolongation of the overall treatment time [5-9-11].

Based on above discussion, the interruption in 
treatment schedule has a negative effect on disease control, therefore this research was planned to investigate a comprehensive study on reasons of radiotherapy interruption at Golestan Hospital which is the major center of radiotherapy and oncology in southwest of Iran.

\section{Materials and Methods}

Cancer patients treated at the Ahvaz Golestan Hospital during March 2012 to March 2013 were included in this retrospective analytical-descriptive study which was done at 2015. All patients treated with Varian Clinac 2100C or Siemens Primus linear accelerators. Patients were included whether their radiation treatment was curative or palliative. Patients treated with no treatment gap were excluded from the study. Interruptions in treatment were considered as the number of calendar days over which it would have been possible to have treatment (i.e., weekdays). Weekends were not regarded as missed appointments. The required information in the questionnaire included age, gender, site of cancer (head and neck v/s other types), intent of treatment (curative $\mathrm{v} / \mathrm{s}$ palliative), interruption period, causes of interruption, patients' address and phone numbers.

The Statistical Package for the Social Sciences (SPSS) version 15 was used to analyze the obtained data. The descriptive statistics were average and standard deviation in quantitive variables, and frequency and percent in qualitative variables. In order to found any relation between different variables, the Fischer, independent $\mathrm{T}$, Chi score and regression logistic tests were applied. P-values of $<0.05$ were considered significant.

\section{Results}

Among 1476 patients of this study, 861 were female $(58.3 \%)$ and 615 were male (41.7\%). About $32.7 \%$ of male patients (201 from 861 patients) and $26.8 \%$ of female

Table 1. Patient Characteristics and Study Variables

\begin{tabular}{lcc}
\hline Variable & Number & Percent \\
\hline Sex & & \\
$\quad$ Male & 615 & $41.7 \%$ \\
$\quad$ Female & 861 & $58.3 \%$ \\
Site of Cancer & & \\
$\quad$ Head and Neck & 376 & $25.5 \%$ \\
$\quad$ Other Sites & 1100 & $74.5 \%$ \\
Living Place & & \\
$\quad$ Ahvaz City & 568 & $38.5 \%$ \\
$\quad$ Khuzestan Province but not in Ahvaz city & 738 & $50 \%$ \\
$\quad$ Out of Khuzestan Province & 170 & $11.5 \%$ \\
Intent of Treatment & & \\
$\quad$ Curative & 1302 & $88.2 \%$ \\
$\quad$ Palliative & 174 & $11.7 \%$ \\
Radiotherapy Interruption & & \\
$\quad$ No Interruption & 1044 & $70.7 \%$ \\
Interruption & 432 & $29.3 \%$ \\
\hline$\quad$ & & \\
\hline
\end{tabular}

Table 2. Causes of Radiotherapy Interruption

\begin{tabular}{lcc}
\hline Interruption Cause & Number & Percent \\
\hline Equipment damages and/or maintenance & 127 & 29.5 \\
Side effects & 73 & 16.7 \\
Public holidays & 35 & 8.1 \\
Patient death & 23 & 5.3 \\
Distance & 20 & 4.7 \\
Treatment cost & 8 & 1.9 \\
Other causes & 34 & 7.9 \\
Unspecified causes & 112 & 25.8 \\
Total & 432 & 100.0 \\
\hline
\end{tabular}

patients (231 from 615 patients) had interruption in their radiotherapy course (Table 1 ). There was no significant correlation between patients' gender and interruption probability (P-value: 0.055, OR: 1.254).

The patients' ages were in the range of 1 to 96 years old with the average age of 51.72 years with 18.004 standard deviation. In 1044 patients without interruption and 432 interrupted patients, average ages were 51.87 and 51.22 years old, respectively. There was no significant relation between patients' ages and interruption probability (P-value: 0.535).

Based on the cancer site, the patients were divided in two categories; 376 patients $(25.5 \%)$ had head and neck cancer and the remaindered 1100 patients (74.5\%) had cancers of other sites. More than $35.1 \%$ of 376 patients with head and neck cancer (132 patients) and $27.3 \%$ of 1100 patients with other sites of cancer $(300$ patients) had interruption in their treatment courses. The probability of treatment interruption in head and neck cancers was significantly higher than other cancers (P-value: 0.014, OR: 1.377).

Also, the patients were categorized for their living place as follows: 568 patients (38.5\%) were in Ahvaz city, 738 patients $(50 \%)$ in Khuzestan province but not in Ahvaz city, and the remaindered 170 patients $(11.5 \%)$ out of Khuzestan province. About $30.3 \%$ of 568 Ahvazi patients (172 patients), $26.5 \%$ of 738 non-Ahvazi patients from Khuzestan province (196 patients), and $37.9 \%$ of 170 patients from out of Khuzestan province (64 patients) had interruption in their treatment courses. There were no statistically significant differences in interruption probability among Ahvazi and non-Ahvazi patients from Khuzestan province (P-value: 0.134). However; the probability of treatment interruption in patients from out of Khuzestan province was significantly higher than Ahvazi (P-value: 0.093, OR: 1.362) and non-Ahvazi patients from

Table 3. Periods of Radiotherapy Interruption

\begin{tabular}{lcc}
\hline Interruption Period & Number & Percent \\
\hline Non & 1044 & 70.7 \\
1-3 days & 195 & 13.2 \\
4-30 days & 165 & 11.2 \\
$>30$ days & 72 & 4.9 \\
Total & 1476 & 100.0 \\
\hline
\end{tabular}


Khuzestan province (P-value: 0.006, OR: 1.642).

Moreover, based on the intent of treatment, the patients were divided into two groups; 1302 patients had curative treatment $(88.2 \%)$ and 174 patients had palliative treatment $(11.7 \%)$

There was no any interruption in $70.7 \%$ of patients (1044 people) in the treatment course (Table 1 and 2). On the other hand, the treatment interruption was found in $29.3 \%$ of patients (432 people), $13.2 \%$ of patients (195 people) with 1 to 3 days interruption period, $11.2 \%$ of patients (165 people) with 4 to 30 days interruption period, and $4.9 \%$ of patients ( 72 people) with more than 30 days interruption period (Table 3 ).

The most common cause of interruption in the treatment course was equipment damages and/or maintenance with $29.5 \%$ (127 patients) of all causes. Other causes of treatment interruption were treatment side effects and general conditions (16.7\%), public holidays $(8.1 \%)$, patients' death $(5.3 \%)$, trip difficulties $(4.7 \%)$, treatment cost $(1.9 \%)$, and miscellaneous causes (shifting to another center and personal problems or tendencies in $7.9 \%)$. In 112 patients (25.8\%) no clear reason was found and the patients were not accessable for more investigation (Table 2).

\section{Discussion}

More than $35.1 \%$ of patients with head and neck cancer and $27.3 \%$ of patients with other sites of cancer had interruption in their treatment courses, which this difference was statistically significant (P-value: 0.014, OR: 1.377). This is in contrast with a Spanish study by Garau et al in 2008 in which only $23.4 \%$ of patients finished their radiotherapy in the planned overall treatment time (OTT) but $48.9 \%$ of head and neck cancer patients finished their treatment in the planned OTT. One of the main reasons for this difference is that for the head and neck cancers, the patients receive more doses of radiation due to curative and radical intent of treatment in these patients, hence showing more severe and debilitating treatment side effects. Also in general, these patients have low socioeconomic status with less social and family support leading to increasing rates of interruption. The Royal College of Radiographers [12-13] recommends that proactive support from a multidisciplinary team of healthcare professionals and counselors be available to patients to reduce treatments missed from acute toxicities. Unfortunately we don't have this team at our center.

About $30.3 \%$ of Ahvazi patients, $26.5 \%$ of non-Ahvazi patients from Khuzestan province, and $37.9 \%$ of patients from out of Khuzestan province had interruption in their treatment courses. The probability of treatment interruption in patients from out of Khuzestan province was significantly higher than Ahvazi (P-value: 0.093, OR: 1.362) and non-Ahvazi patients from Khuzestan province (P-value: 0.006, OR: 1.642). This difference is most likely due to the distance of living place to the radiotherapy center.

The treatment interruption was found in $29.3 \%$ of patients, $13.2 \%$ of patients with 1 to 3 days gap, $11.2 \%$ of patients with 4 to 30 days gap, and $4.9 \%$ of patients with more than 30 days gap. This rate was equal or lower than other centers. A study by Barton et al in 1990 showed that 589 out of $1012(58 \%)$ patients with laryngeal cancer had radiotherapy interruption at Princess Margaret Hospital of Toronto during 22 years period from 1960 to 1982 [14]. A national audit of radiotherapy in head and neck cancer in the United Kingdom by James et al in 2000 showed that 1467 out of 2553 (55\%) patients had one or more treatment interruptions [15]. Another audit of the effectiveness of national radiotherapy practice in head and neck cancer in the United Kingdom by James et al in 2005 showed that 397 out of $631(63 \%)$ patients had one or more treatment interruptions [16]. A study by Gidding et al in 2009 [4] showed that $74 \%$ of 471 patients with head and neck cancer had radiotherapy interruption at the Vancouver Cancer Centre of the BC Cancer Agency during 3 years period from 2006 to 2008. Treatment breaks of greater than three days were present in $11 \%$ of radiotherapy courses. A Spanish study by Garau et al in 2008 [12] showed that $76.6 \%$ of 478 cancer patients with all primary sites treated with curative intent had radiotherapy interruption and $17.9 \%$ of the interruptions were greater than 5 days and $5.6 \%$ greater than 10 days.

There are some differences in the causes of interruption in Ahvaz and those reported in other centers. Public holidays are one of the most common causes of treatment gaps; however in Ahvaz holidays accounted for $8.1 \%$ of treatment interruptions, whereas in the United Kingdom they were responsible for $39 \%$, in the Spanish study $45 \%$, and in Vancouver for $69 \%$ of treatment interruptions. This is because of efforts aimed at working our staff and machines in the most public holidays in Ahvaz.

Machine breakdown and maintenance account for $44 \%$ of treatment interruptions in the United Kingdom, $45 \%$ in Spain, and 2\% in Vancouver. About $29.4 \%$ of gaps were due to this reason in Ahvaz. This is because of efforts aimed at treating patients with another machine, despite of high rate of machine break down at our center.

The second most common cause of interruption in Ahvaz was treatment side effect $(16.7 \%)$ which was higher than other centers and equal to older studies like the study of Barton et al in which radiation side effects was responsible for $16 \%$ of all treatment breaks. This rate was $2 \%$ in Spain, $10 \%$ in Vancouver, and $8 \%$ in the United Kingdom. This is because of lack of a multidisciplinary team to support patients to reduce treatments missed from acute toxicities.

In conclusions, unplanned radiotherapy interruption is a major problem in normal clinical practice. Generally, treatment interruption at our center was not much higher than other centers; however, the most common cause of treatment interruption was equipment damages and/or maintenance, which was higher than most centers. This important issue requires special attention of authorities. Most interruptions are short and can be planned by treating patients on weekends and holidays to maintain the OTT. 


\section{Acknowledgments}

This article is retrieved from undergraduate thesis No. ein-1037 of Ahvaz Jundishapur University of Medical Sciences.

\section{Authors' contributions}

S.R have conceived the manuscript and revised it. N.H., M.B., S.A, M.H. , H.S. and S.T. . provided clinical data, pathological diagnoses, and information. also wrote the manuscript and performed the technical tests.

\section{Compliance with ethical guidelines}

\section{Conflict of interest}

Authors declare that they have no conflict of interest.

\section{Funding}

This article is retrieved from undergraduate thesis No. ein-1037 of Ahvaz Jundishapur University of Medical Sciences.

\section{Human and animal rights and informed consent}

All the procedures performed in the studies involving human participants were in accordance with the ethical standards of local ethics committee of the Ahvaz Jundishapur University of Medical Sciences (IR.AJUMS. REC.1392.209), as well as 1964 Helsinki declaration. Written informed consent was obtained from all patients and normal subjects.

\section{References}

1. Symonds RP, Foweraker K. Principles of Chemotherapy and Radiotherapy. Curr Obstet Gynaecol. 2006;16(2):100-106.

2. Sridhar T, Symonds RP. Principles of Chemotherapy and Radiotherapy. Obstet Gynaecol Repro Med. 2009;19(3):6167.

3. Sinclair JA, Oats JP, Dale RG. BED-Time Charts and Their Application to the Problems of Interruptions in External Beam Radiotherapy Treatments. Int J Radiat Oncol. 1999;44(2):381-389.

4. Giddings A. Treatment Interruptions in Radiation Therapy for Head-And-Neck Cancer: Rates and Causes. J Med Imaging Radiat Sci. 2010;41:222-229.

5. Bese NS, Hendry J, Jeremic B. Effect of Prolongation of Overall Treatment Time Due to Unplanned Interruptions During Radiotherapy of Different Tumor Sites and Practical Methods for Compensation. Int J Radiat Oncol. 2007;68(3):654-661.

6. Withers HR, Maciejewski B, Taylor JM, Hliniak A. Accelerated Repopulation in Head and Neck Cancer. Front Radiat Ther Oncol. 1988;22:105-110.

7. Bentzen SM, Thames HD. Clinical Evidence for Tumor Clonogen Regeneration: Interpretations of the Data. Radiother Oncol. 1991;22(3):161-166.

8. Fesinmeyer MD, Mehta V, Blough D, Tock L, Ramsey SD. Effect of Radiotherapy Interruptions on Survival in Medicare Enrollees with Local and Regional Head and Neck Cancer. Int J Radiat Oncol. 2010;78(3):675-681.

9. Hendry JH, Mackay RI, Roberts SA, Slevin NJ. Outstanding issues in radiation dose-fractionation studies. Int $\mathrm{J}$ Radiat Biol. 1998;4:383-394.
10. Yaes RJ. Some common causes of treatment prolongation. Int J Radiat Oncol Biol Phys. 1995;31:686-687.

11. Lindberg RD, Jones K, Garner HH, Jose B, Spanos Jr WJ et al. Evaluation of unplanned interruptions in radiotherapy treatment schedules. Int J Radiat Oncol Biol Phys. 1988;14:811-815.

12. Garau MM, Monné JS, Serés MJC, Binefa CM, Llopis MP. Compliance to the prescribed overall treatment time (OTT) of curative radiotherapy in normal clinical practice and impact on treatment duration of counteracting short interruptions by treating patients on Saturdays. Clin Transl Oncol. 2009;11:302-311.

13. Board of Faculty of Clinical Oncology. The Royal College of Radiographers (2008). The timely delivery of radical radiotherapy: standards and guidelines for the management of unscheduled treatment interruptions, third edition. http:// www.rcr.ac.uk

14. Barton MB, Keane TJ, Gadalla T, Maki E. The Effect of Treatment Time and Treatment Interruption on Tumor Control Following Radical Radiotherapy of Laryngeal Cancer. Radiother Oncol. 1992;23:137-143.

15. James ND, Robertson G, Squire CJ, Forbes H, Jones K at al. A National Audit of Radiotherapy in Head and Neck Cancer. Clin Oncol. 2003;15:41-46.

16. James ND, Williams MV, Summers ET, Jones K, Cottier B. The Management of Interruptions to Radiotherapy in Head and Neck Cancer, an Audit of the Effectiveness of National Guidelines. Clin Oncol. 2008;20:599-605.

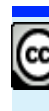

This work is licensed under a Creative Commons AttributionNon Commercial 4.0 International License. 